\title{
PATOlogías Posparto y ORIGEN DE VACAS INFÉRTILES EN GANAdo LECHERO EN EL ALtiplano MEXICANO
}

\author{
POSTPARTUM PATHOLOGIES AND ORIGIN OF INFERTILE COWS IN DAIRY \\ CATtLe in the MeXicAn HighlandS
}

\section{Miguel A. Lammoglia-Villagómez ${ }^{1}\left(\mathbb{C}\right.$, Javier C. Huerta-Peña*1 ${ }^{*}$ y Pablo R. Marini $^{2}$ (10}

\author{
${ }^{1}$ Universidad Veracruzana, Facultad de Ciencias Biológicas y Agropecuarias, Poza Rica-Tuxpan. \\ ${ }^{2}$ Centro Latinoamericano de Estudios de Problemáticas Lecheras (CLEPL). Facultad de Ciencias Veterinarias-Universidad Na- \\ cional de Rosario, Provincia de Santa Fe, Argentina.
}

*Autor para correspondencia: jhuerta@uv.mx

\begin{abstract}
Resumen
La infertilidad en vacas lecheras estabuladas es un problema creciente y una de las principales causas de desecho. El objetivo fue determinar el origen de infertilidad en vacas lecheras estabuladas del altiplano mexicano con patologías posparto. Se estudiaron 1,110 vacas Holstein estabuladas y divididas por número de parto: primero (n=389); segundo, ( $n=296)$ y tres o más $(n=425)$. El análisis estadístico se realizó haciendo una comparación de medias (modelo ANOVA) y factores de riesgo (Odds Ratio). El 80.5\% de las vacas permanecieron sanas después del parto, 15,6\% resultaron infértiles y el 3,9\% no presentó cambios. La prevalencia de patologías fue del 19,5\% encontrando que el grupo de vacas con patologías reproductivas tuvieron mayor porcentaje $(\mathrm{p}<0,05)$ de infertilidad $(25,0 \%)$. Se incrementó el problema de infertilidad con el número de partos, siendo el grupo de vacas de tres o más partos y con patologías reproductivas las de mayor porcentaje de vacas infértiles comparadas con las de segundo y primer parto (39,0\%, 30,0\% y 14,0\%; $\mathrm{p}<0,05$, respectivamente). Se identificaron como factores de riesgo vacas de segundo parto (OR: 2,24 IC95\%: 1,06-4,95) y aquellas con más de tres partos que presentaron patologías reproductivas (OR: 1,95 IC95\%: 1,03-3,71). En conclusión, las vacas que presentaron patologías reproductivas tuvieron el mayor porcentaje de infertilidad, el cual incrementó hasta cuatro veces el riesgo de quedar vacías, especialmente si presentaban más de dos partos. No se pudo explicar por completo el porcentaje de vacas infértiles con las patologías posparto, quedando un $15.6 \%$ de vacas infértiles con diferente origen.
\end{abstract}

Palabras clave: Infertilidad, problemas metabólicos, vacas lecheras, vacas estabuladas, altiplano. 


\begin{abstract}
Infertility in dairy cows under intensive productive systems is a growing problem and is one of the main causes of culling. The objective was to determine the origin of infertility in dairy cows under intensive productive systems from the Mexican highlands with postpartum pathologies. In this experiment 1,110 housed Holstein cows were studied. Cows were divided by calving number: First $(n=389)$; Second $(n=296)$ and three or more $(n=425)$. Statistical analysis: comparison of means (Anova model) and univariate analysis. High percentage of the cows remained healthy after calving (80.5\%); however, $15.6 \%$ of these cows were infertile. The prevalence of pathologies was $19.5 \%$, and $26.3 \%$ of these cows were infertile. Cows with reproductive pathologies had a higher percentage $(p<0.05)$ of infertile cows $(25.0 \%)$. The infertility increased with the number of calvings, cows with three or more calvings and with reproductive pathologies had the highest percentage of infertile cows compared with those of second and first calving ( $39.0 \%$, $30.0 \%$ and $14.0 \%$, respectively). Second calving cows (OR: $2.2495 \%$ CI: 1.06-4.95) and cows of three or more calvings and that presented reproductive pathologies (OR: $1.9595 \%$ CI: 1.03-3.71) were identified as risk factors. In conclusion, cows that presented reproductive pathologies had the highest percentage of infertile cows and the risk of remaining infertile increased up to 4 times, especially if they presented more than 2 calvings. The percentage of infertile cows with postpartum pathologies could not be fully explained, leaving $15.6 \%$ of infertile cows with different origins.
\end{abstract}

Keywords: Infertility, metabolic problems, dairy cows, housed cows, highlands.

Forma sugerida de citar: Lammoglia-Villagómez, M.A., Huerta-Peña, J.C. y Marini, P.R. (2021). Patologías Posparto y Origen de Vacas Infértiles en Ganado Lechero en el Altiplano Mexicano. La Granja: Revista de Ciencias de la Vida. Vol. 33(1):44-52. http://doi.org/10.17163/lgr. n33.2021.04.

IDs Orcid:

Miguel A. Lammoglia-Villagómez: http:/ / orcid.org/0000-0002-2958-0518

Javier C. Huerta-Peña: http:/ / orcid.org/0000-0001-5007-1032

Pablo R. Marini: http:/ / orcid.org/0000-0002-2958-0518 


\section{Introducción}

Los altos rendimientos de producción de leche en los últimos 70 años en ganado lechero especializado han sido el resultado de una intensa selección genética (Oltenacu y Algers, 2005; Miglior y col., 2017) y últimamente con ayuda de la evaluación genómica de los toros jóvenes. Sin embargo, hoy en día, la selección genética ha tenido su precio ya que las vacas altamente productoras han disminuido drásticamente su fertilidad (Diskin, Murphy y Sreenan, 2006; Sheldon y col., 2009a; Sheldon y col., 2009b); así también, se han incrementado los problemas de salud durante las primeras semanas posparto (LeBlanc, 2010). No obstante, a pesar de los progresos en el cuidado en la ganadería lechera en la etapa de conversión existen todavía problemas de salud (Esposito y col., 2014). LeBlanc (2010) reportó una gran prevalencia de padecimientos posparto concluyendo que una de cada dos vacas lecheras presentaba alguna patología, por lo que el aumento de enfermedades tendrá repercusiones negativas en la reproducción (Barker y col., 1998; Lammoglia y col., 2015; Sheldon, 2020). Las vacas que presentan alguna patología posparto, ya sea de índole metabólico (cetosis, hipocalcemia, desplazamiento de abomaso, etc.), o inmunológico (retención de placenta, metritis, mastitis, etc) tuvieron una menor producción, así como una menor tasa de fertilidad y se incrementó el riesgo de ser descartadas del hato (Walsh y col., 2007; Dubuc y col., 2012; Hudson y col., 2012; Vieira-Neto y col., 2014; Denis-Robichaud y Dubuc, 2015; Vallejo-Timarán y col., 2017).

Se ha identificado que las consecuencias de las patologías reproductivas en el posparto también son a mediano plazo, ya que afectan la fertilidad en los primeros servicios e incrementan el periodo abierto y el descanso entre partos; estos aspectos resultan altamente costosos para cualquier sistema de producción, principalmente en la ganadería intensiva. Sheldon (2020) encontraron que parte de la infertilidad se originaba durante la infección del útero, ya que las bacterias, toxinas y lipopolisacáridos podían llegar al ovario, utilizando como transporte la circulación del sistema pedículo vascular úteroovárico. Una vez en el ovario desencadenan un proceso inflamatorio que puede durar semanas o incluso meses, ocasionando ooforitis (daño al cuerpo lúteo) al ovocito y en general disfunción ovárica. El objetivo de la investigación fue determinar el origen de infertilidad en vacas lecheras estabuladas del altiplano mexicano con patologías posparto.

\section{Materiales y Métodos}

El estudio se llevó a cabo en una finca pecuaria de ganado lechero especializado. Se encuentra ubicada en el altiplano mexicano, en el estado de Hidalgo, México $\left(24^{\circ} \mathrm{N} 103^{\circ} \mathrm{O}, 24^{\circ} \mathrm{N} 103^{\circ} \mathrm{O}\right)$ a una altitud de 1980 msnm, con un clima semi seco templado, con temperatura media de $14,5^{\circ} \mathrm{C}$, temperatura (máxima de $24,4^{\circ} \mathrm{C}$ y temperatura mínima de $5,3^{\circ} \mathrm{C}$ ), humedad relativa media de $65,6 \%$, (humedad relativa máxima de $93,5 \%$ y humedad relativa mínima de $30,4 \%)$.

\subsection{Preparación de Catalizadores}

La unidad de producción pecuaria cuenta con 1058 vacas Holstein en ordeño y un promedio en línea de 36,5 \pm 1,5 L/vaca/día. Las vacas se encuentran estabuladas y son ordeñadas tres veces al día y ocurre un promedio de $110 \pm 15$ partos por mes.

El manejo que se llevó a cabo con las vacas fue de la siguiente manera: las vacas después del parto se ordeñaron tres veces al día y se mantuvieron 21 días en un corral de recién paridas bajo condiciones de alto nivel de confort (naves frescas, echaderos individuales y amplios $\left( \pm 7 \mathrm{~m}^{2}\right)$, arena limpia y abundante, agua fresca a libre acceso, área de ejercicio con piso de tierra $\left( \pm 8 \mathrm{~m}^{2}\right)$, y una baja población de vacas por corral $( \pm 25))$ en una etapa de transición que consistió en la misma ración integral preparto (Alfalfa $87 \%$, maíz $88 \%$, silo avena $44 \%$ ), sin sales aniónicas, y posteriormente fueron integradas a los corrales de reto de alta producción.

Durante 10 días consecutivos posteriores al parto se les tomó diariamente la temperatura rectal y se llevaron registros como indicador de un estado de salud idóneo. Las vacas se agruparon de acuerdo a los padecimientos que presentaron del día 0 a los 45 días posparto. Los diagnósticos y tratamientos de cada padecimiento fueron realizados por un clínico especialista en bovinos lecheros.

Se obtuvo la información de 1110 vacas del programa Dairy-Com $305^{\circledR}$ software administrativo para establos lechero, quedando distribuidas en vacas 
de uno, dos y tres o más partos; asimismo, se clasificaron como sanas, con problemas reproductivos, alimentarios, metabólicos, locomotores y sanitarios (Tabla 1).

Tabla 1. Categorización de la información de animales por número de parto y estado de salud.

\begin{tabular}{ccccc}
\hline Categorías & $\begin{array}{c}\text { Primer } \\
\text { Parto }\end{array}$ & $\begin{array}{c}\text { Segundo } \\
\text { Parto }\end{array}$ & $\begin{array}{c}\text { Tres o Más } \\
\text { Partos }\end{array}$ & Total De Vacas \\
\hline Sanas & 309 & 254 & 330 & 893 \\
Reproductivo & 64 & 20 & 44 & 128 \\
Alimentario & 4 & 5 & 8 & 17 \\
Metabólico & 3 & 1 & 10 & 14 \\
Locomotor & 3 & 6 & 10 & 19 \\
Sanitario & 6 & 10 & 23 & 39 \\
Total & 389 & 296 & 425 & 1110 \\
\hline
\end{tabular}

Debido al bajo número de animales en las diferentes categorías, excepto problemas reproductivos, se unieron en una categoría denominada "otros" para que el análisis estadístico tuviera un mayor número de vacas dentro de esta variable; sin embargo, algunas características como condición corporal, condición de la salud post-parto o nivel de producción láctea no fueron evaluadas durante la investigación debido a que no era de interés en esta investigación.

El análisis estadístico consistió en la comparación de medias utilizando ANOVA con la prueba de Tukey y análisis multivariado utilizando el software Statistica versión $10^{\circledR}$. La determinación de riesgo se calculó con Odds Ratio (OR) con el programa Win Episcope Ver. 2.0 ${ }^{\circledR}$ (Thrusfield y col., 2001) donde se consideró la interpretación de las características de riesgo de acuerdo al número de partos, así como de las categorías con intervalos de confianza del 95\% (IC95\%).

\section{Resultados y Discusión}

\subsection{Resultados}

De un total de 1110 vacas se encontró una prevalencia del 19,5\% con problemas de salud después del parto. Las hembras con presencia de patologías después del parto son las de porcentajes de fertilidad más bajos $(p<0,05)$ que las vacas que no enfermaron (Tabla 2). Las vacas que presentaron patologías reproductivas posterior al parto, como retención de membranas fetales y/o metritis, mostraron una fertilidad más baja $(p<0.05)$ en comparación con otros padecimientos (Tabla 2). El número de partos por sí solo afectó $(\mathrm{p}<0.05)$ la fertilidad de las vacas sanas. Las vacas primíparas tuvieron la mejor fertilidad y las vacas de tres o más partos tuvieron la fertilidad más baja de todos los grupos (Figura 1).

Tabla 2. Fertilidad $(\%)$ de las vacas afectada $(p<0,05)$ por patologías posparto y por el número de parto de vacas especializadas en leche.

\begin{tabular}{ccccc}
\hline \multirow{2}{*}{ Condición de las vacas } & \multicolumn{4}{c}{ Número de partos } \\
& Uno & Dos & Tres o más & $p<0,05$ \\
\hline Sanas & $92,2^{a}$ & $85,8^{b}$ & $76,0^{c}$ & 0,05 \\
Patologías reproductivas & $86,0^{a}$ & $70,0^{b}$ & $61,0^{c}$ & 0,05 \\
Otros & $87,5^{a}$ & $59,0^{b}$ & $72,5^{c}$ & 0,05 \\
\hline
\end{tabular}


Las patologías reproductivas afectaron la fertilidad de las vacas $(p<0,05)$. Las vacas de primer parto que presentaron patologías reproductivas posparto tuvieron $6,2 \%$ menos $(p<0,05)$ fertilidad en relación a las vacas sanas; así mismo, las vacas de dos partos y las vacas de tres o más que tuvieron patologías reproductivas después del parto presentaron $15 \%$ menos fertilidad que las vacas que permanecieron sanas. No obstante, las patologías reproductivas tuvieron un efecto menor $(p<0,05)$ en la fertilidad de las vacas de primer parto comparadas con las vacas de dos y tres o más partos (Figura 2).
Los resultados del análisis univariados para determinar factores de riesgo mostraron que las vacas de tres o más partos que presentan patologías reproductivas tuvieron hasta cuatro veces mayor riesgo (OR 1,95\%; IC95\% 4) de permanecer vacías (Tabla 3) y que las vacas de más de dos partos con hasta cinco veces menos posibilidades de quedar gestantes (OR 2,24\%; IC95\% 5; Tabla 3). Se encontró que los padecimientos en los primeros 30 días posparto incrementan en mayor grado la infertilidad en vacas primerizas, de segundo parto y de tres o más partos, aunque esto no explican en su totalidad las causas de infertilidad.

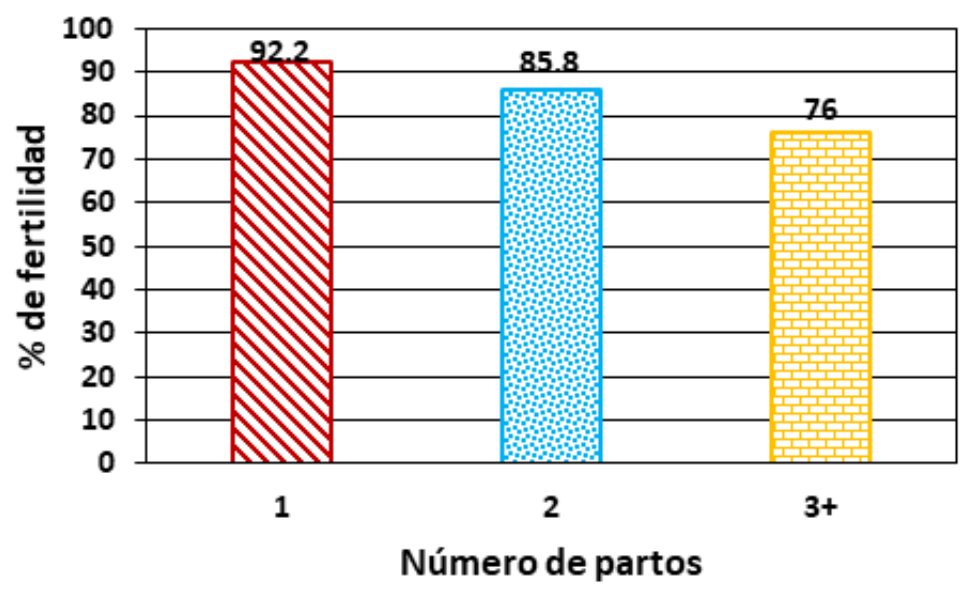

Figura 1. Efecto del número de parto en la fertilidad de vacas especializadas en leche.

\subsection{Discusión}

Con los resultados se puede deducir que la prevalencia de las patologías posparto del establecimiento lechero analizado del altiplano mexicano fue del $19,5 \%$. Es decir, que una de cada cinco vacas sufrió alguna patología en el período posparto. Estos resultados son menores a lo reportados por LeBlanc (2010) en donde la prevalencia de padecimientos en el posparto fue una de cada dos vacas lecheras con alguna patología. Dubuc y Denis-Robichaud (2017) en 126 rodeos lecheros encontraron una prevalencia de enfermedades posparto de 0 a $80 \%$, y plantearon la necesidad de una investigación a nivel de rebaño debido a la variación tan amplia de la prevalencia de la enfermedad posparto entre rebaños. También la prevalencia de patologías posparto encontrada en este estudio fue menor a la reportada por Santos y col. (2010) en un estudio con 5179 vacas, donde encontraron el $44,2 \%$ de vacas con patologías. Las patologías posparto son ocasionadas por múltiples factores que inician desde el periodo seco hasta el periodo de frescas (Chebel, Mendonça y Baruselli, 2018). Por ejemplo, una pérdida de condición corporal en el periodo seco puede tener un efecto marcado en la salud durante el posparto (Chebel, Mendonça y Baruselli, 2018). Cabe resaltar que en vacas lecheras el estro es más prolongado en época de invierno mientras que en verano suele ser menor, lo que dificulta la correcta detección de estros. Esta característica se desencadena por el estrés térmico que sufren las vacas lecheras, en especial las de raza tipo Holstein (Castaño y col., 2014). Es posible que en este estudio la prevalencia de patologías posparto fue menor que los resultados publicados por otros autores, debido a la diferencia en el manejo de las vacas que pudieron iniciar desde el periodo seco hasta el periodo de frescas. 


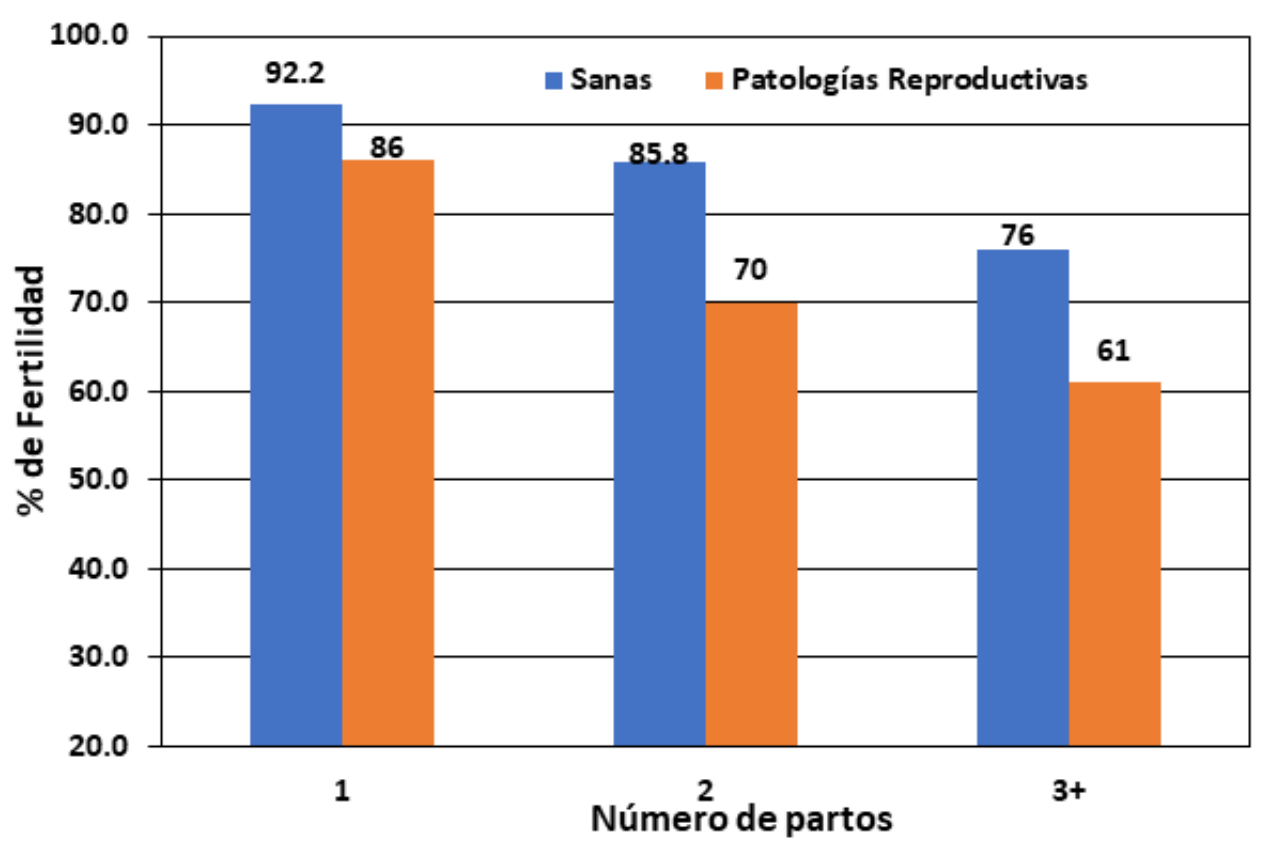

Figura 2. Efecto de las patologías reproductivas posparto en la fertilidad de vacas especializadas en leche en el altiplano mexicano.

Los resultados de la investigación indican que el número de partos de las vacas afectó la prevalencia. Las vacas primíparas presentaron menor prevalencia. Estos resultados son corroborados por Wittrock y col. (2011) quienes reportaron una menor incidencia de patologías posparto en vacas primíparas que multíparas. La menor prevalencia de patologías posparto en estas vacas puede deberse a que las vacas de primer parto presentan una mejor adaptación metabólica y menor pérdida de condición corporal en la transición que las vacas de dos o más partos y esto pudiera contribuir a las diferencias (Adrien y col., 2012; Wathes y col., 2007). Entre las causas principales de infertilidad en el ganado bovino se encuentran los desórdenes genéticos, entre los que se pueden mencionar las anormalidades cromosómicas, malformaciones congénitas o freemartinismo; de igual forma, los factores hormonales dan como resultado la incapacidad cíclica de las hembras (Córdova y col., 2002).

Los problemas reproductivos durante el periodo posparto, ya sea retención de membranas fetales, metritis o la combinación de ambas patologías, afectaron el porcentaje de vacas gestantes independientemente del número de partos. Estos resultados son confirmados por Melendez y col. (2009) y Deori y Phookan (2015) quienes mencionan que las patologías reproductivas posparto redujeron la fertilidad en las vacas lecheras. También Santos y col. (2010) encontraron que hembras que presentan metritis o endometritis posparto tuvieron menor fertilidad que las vacas sanas. Sheldon (2020) encontraron una posible explicación de la causa fisiológica al mencionar que debido a que las vacas tienen una circulación única entre el útero y los ovarios (sistema pedículo vascular útero-ovárico), tiene una relación estructural de la irrigación arterial y venosa muy importante en el establecimiento de los mecanismos fisiológicos coordinados del útero y los ovarios. En el área de aposición entre la arteria ovárica y la vena uterina existe una disminución del espesor de las paredes de las mismas, aunque no se ha demostrado conexión vascular directa entre arteria y vena, y es aquí donde se realiza el intercambio de moléculas como la prostaglandina $F 2 \alpha$, la cual pasa del sistema venoso uterino al sistema arterial ovárico.

Existen dos mecanismos para realizar el intercambio de moléculas; el primero es la vía de difusión a través de la red de vasos linfáticos úteroovárico (los vasos linfáticos se adhieren estrechamente a la red de vasos venosos y arteriales en el ligamento ancho del ovario) y el segundo por proteínas transportadoras. De esta manera, las bacte- 
rias, sus toxinas y lipopolisacáridos alcanzan a llegar al ovario donde producen una reacción inflamatoria que interfiere con la salud de los ovocitos y la producción de hormonas, tanto del cuerpo lúteo como de los folículos, afectando así la fertilidad de las vacas (Sheldon, 2020; Fabian y col., 2010; Chebel, 2007).

Tabla 3. Riesgo de infertilidad por categorías de patologías posparto asociado al número de partos en vacas especializadas.

\begin{tabular}{lll}
\hline Primer Parto & OR & $I C_{95 \%}$ \\
\hline Sanas gestantes & 1,98 & $0,93-4,20$ \\
Sanas vacías & 0,51 & $0,24-1,08$ \\
Problemas reproductivos (Gestantes) & 0,51 & $0,23-1,14$ \\
Problemas reproductivos (Vacías) & 1,96 & $0,88-4,37$ \\
Otros problemas (Gestantes) & 0,66 & $0,14-3,00$ \\
Otros problemas (Vacías) & 1,52 & $0,33-6,92$ \\
\hline Segundo Parto & $\mathbf{O R}$ & $I C_{95}$ \\
\hline Sanas gestantes & $\mathbf{2 , 2 4}$ & $\mathbf{1 , 0 6 - 4 , 9 5}$ \\
Sanas vacías & 0,45 & $0,20-0,98$ \\
Problemas reproductivos (Gestantes) & 0,40 & $0,15-1,08$ \\
Problemas reproductivos (Vacías) & 2,48 & $0,92-6,64$ \\
Otros problemas (Gestantes) & 0,59 & $0,18-1,87$ \\
Otros problemas (Vacías) & 1,70 & $0,53-5,41$ \\
\hline Tercer Parto & $\mathbf{O R}$ & $I C_{95}$ \\
\hline Sanas gestantes & 1,54 & $0,94-2,53$ \\
Sanas vacías & 0,65 & $0,40-1,07$ \\
Problemas reproductivos (Gestantes) & 0,51 & $0,27-0,97$ \\
Problemas reproductivos (Vacías) & $\mathbf{1 , 9 5}$ & $\mathbf{1 , 0 3 - 3 , 7 1}$ \\
Otros problemas (Gestantes) & 0,91 & $0,47-1,76$ \\
Otros problemas (Vacías) & 1,10 & $0,57-2,12$ \\
\hline
\end{tabular}

El porcentaje de vacas gestantes también fue afectado por el número de partos, en donde las vacas con mayor número de partos estuvieron más propensas a quedar vacías, sin importar si presentaron problemas de salud durante el posparto. Estos resultados son corroborados por otros estudios publicados por Lucy, Butler y Garverick (2014), quienes relacionan la producción láctea en hembras de más partos; es decir, al producir más leche es común encontrar un mayor número de estas vacas con balance energético negativos y con elevadas concentraciones tanto de $\beta$-hidroxibutirato como de ácidos grasos no esterificados. El balance energético negativo en el postparto de la vaca lechera se asocia a una reducción en los pulsos de GnRH y LH, afectando el crecimiento, maduración y ovulación del folículo, resultando en una baja de fertilidad (Crowe, Diskin y Williams, 2014). Lo que explicaría, por qué no todas las vacas que se mantuvieron sanas durante el posparto quedaron gestantes.

De igual manera, la explicación a los resulta- dos de las vacas sanas que quedaron vacías puede deberse a causas ajenas a los problemas de salud como problemas metabólicos (balance energético negativos, incremento en las concentraciones de $\beta$-hidroxibutirato y ácidos grasos no esterificados) que contribuyen a la infertilidad de las vacas sanas. Asimismo, Trevisi y col. (2011) concluyeron que los procesos proinflamatorios subclínicos después del parto en vacas lecheras tienen efectos a largo plazo entre ellos disminuir o incrementar el riesgo de infertilidad en estas vacas.

\section{Conclusiones}

Las patologías reproductivas tuvieron el mayor impacto en la fertilidad e incrementaron el riesgo de que las vacas quedaran vacías, sobre todo en aquellas que presentaban más de dos partos. Aunque, por sí solo, el número de partos afectó la fertilidad e incrementó el riesgo de quedar vacías; sin embargo, no se pudo determinar por completo la infertilidad 
con las patologías posparto. Se recomienda maximizar el cuidado de las vacas en el periodo de transición para lograr reducir la presencia de enfermedades y disminuir los porcentajes de vacas problemas.

\section{Referencias}

Adrien, M. y col. (2012). «Nutritional regulation of body condition score at the initiation of the transition period in primiparous and multiparous dairy cows under grazing conditions: milk production, resumption of post-partum ovarian cyclicity and metabolic parameters». En: Animal 6.2, 292-299. Online:https://bit.ly/3umJLAk.

Barker, A. y col. (1998). "Influence of clinical mastitis during early lactation on reproductive performance of Jersey cows». En: Journal of dairy science 81.5, 1285-1290. Online:https: / / bit.ly / 2NkOke6.

Castaño, F. y col. (2014). «Impacto del estrés calórico sobre la actividad reproductiva en bovinos y consideraciones para mitigar sus efectos sobre la reproducción». En: Revista Biosalud 13.2, 84-94. Online:https:/ / bit.ly/3aF8EQ9.

Chebel, R., L. Mendonça y P. Baruselli (2018). «Association between body condition score change during the dry period and postpartum health and performance». En: Journal of dairy science 101.5, 4595-4614. Online:https : / / bit . ly / $3 \mathrm{kgIOVJ}$.

Chebel, R.C. (2007). «Mastitis effects on reproduction». En: NMC Regional Meeting Proceedings. Recuperado de http: / / www.agweb.com/assets / import/files/repro.pdf, págs. 43-55.

Córdova, A. y col. (2002). «Causas de infertilidad en ganado bovino.» En: Medicina Veterinaria 19.9, 112-124. Online:https://bit.ly/3uly11e.

Crowe, M, M. Diskin y E. Williams (2014). «Parturition to resumption of ovarian cyclicity: comparative aspects of beef and dairy cows». En: Animal 8.s1, 40-53. Online:https: / / bit.ly/3aFvTJW.

Denis-Robichaud, J. y J. Dubuc (2015). «Determination of optimal diagnostic criteria for purulent vaginal discharge and cytological endometritis in dairy cows». En: Journal of dairy science 98.10, 6848-6855. Online:https://bit.ly/3qFr6ha.

Deori, S. y A. Phookan (2015). «Bovine postpartum metritis and its therapeutics: A Review». En: Indian journal of Science and Technology 8.23, 1-5. Online:https:/ / bit.ly/3uuJxaG.
Diskin, M., J. Murphy y J. Sreenan (2006). «Embryo survival in dairy cows managed under pastoral conditions». En: Animal reproduction science 96.34, 297-311. Online:https:/ / bit.ly/37CKIuC.

Dubuc, J. y J. Denis-Robichaud (2017). «A dairy herd-level study of postpartum diseases and their association with reproductive performance and culling». En: Journal of dairy science 100.4, 3068-3078. Online:https://bit.ly/3qCBgyW.

Dubuc, J. y col. (2012). «Risk factors and effects of postpartum anovulation in dairy cows». En: Journal of dairy science 95.4, 1845-1854. Online:https://bit.ly/3siE8kO.

Esposito, G. y col. (2014). «Interactions between negative energy balance, metabolic diseases, uterine health and immune response in transition dairy cows». En: Animal reproduction science 144.3-4, 60-71. Online:https:/ / bit.ly/2MbVReB.

Fabian, D. y col. (2010). «The effect on preimplantation embryo development of non-specific inflammation localized outside the reproductive tract». En: Theriogenology 74.9, 1652-1660. Online:https:/ / bit.ly/3dvlRwJ.

Hudson, C. y col. (2012). "Associations between udder health and reproductive performance in United Kingdom dairy cows». En: Journal of Dairy Science 95.7, 3683-3697. Online:.

Lammoglia, M. y col. (2015). «The effect of the most common diseases during the first 100 days of post partum on pregnancy rate and reproductive performance in the Mexican highlands dairy cows.» En: Research Opinions in Animal and Veterinary Sciences 5.9, 370-374. Online:https: / / bit. ly/3s8JNdg.

LeBlanc, S. (2010). «Monitoring metabolic health of dairy cattle in the transition period». En: Journal of reproduction and Development 56.S, S29-S35. Online:https://bit.ly/2ZEROdT.

Lucy, M., S. Butler y H. Garverick (2014). «Endocrine and metabolic mechanisms linking postpartum glucose with early embryonic and foetal development in dairy cows». En: Animal 8.s1, 82-90. Online:https://bit.ly/3pKcrjk.

Melendez, P. y col. (2009). «Relationship between serum nonesterified fatty acids at calving and the incidence of periparturient diseases in Holstein dairy cows». En: Theriogenology 72.6, 826-833. Online:https://bit.ly/2NiELfJ.

Miglior, Filippo y col. (2017). «A 100-Year Review: Identification and genetic selection of economically important traits in dairy cattle». En: Jour- 
nal of Dairy Science 100.12, 10251-10271. Online:https:/ / bit.ly/3aGLLf7.

Oltenacu, P. y B. Algers (2005). «Selection for increased production and the welfare of dairy cows: are new breeding goals needed?» En: AMBIO: $A$ Journal of the Human Environment 34.4, 311-315. Online:https:/ / bit.ly/2Mees9S.

Santos, J.E. y col. (2010). «Effect of reducing the period of follicle dominance in a timed artificial insemination protocol on reproduction of dairy cows». En: J. Dairy Sci. 93.7, 2976-88. Online: http://bit.ly/3pKDYB6.

Sheldon, I. (2020). «Diagnosing postpartum endometritis in dairy cattle». En: The Veterinary Record 186.3, 88-90. Online:https:/ / bit.ly/2ZApQjx.

Sheldon, I. y col. (2009a). «Defining postpartum uterine disease and the mechanisms of infection and immunity in the female reproductive tract in cattle». En: Biology of reproduction 81.6, 1025-1032. Online:https:/ / bit.ly/3ujwWaa.

Sheldon, I. y col. (2009b). "Mechanisms of infertility associated with clinical and subclinical endometritis in high producing dairy cattle». En: Reproduction in domestic animals 44.S3, 1-9. Online:https:/ / bit.ly/2NPf31X.

Thrusfield, M. y col. (2001). «Win Episcope 2.0: improved epidemiological software for veterinary medicine». En: Veterinary Record 148.18, 567-572. Online:https:/ / bit.ly/3umW5k2.
Trevisi, E. y col. (2011). «Acute Phase Proteins as Early Non-Specific Biomarkers of Human and Veterinary Diseases». En: Istituto di Zootecnica, Universitá Cattolica del Sacro Cuore, Piacenza. Cap. Inflammatory response and acute phase proteins in the transition period of high-yielding dairy cow. Chapter 14, págs. 355-380.

Vallejo-Timarán, D. y col. (2017). «Efecto de las enfermedades en posparto temprano sobre el intervalo parto concepción: estudio de cohorte en vacas lecheras de Pasto, Colombia». En: CES Medicina Veterinaria y Zootecnia 12.1, 33-43. Online:https://bit.ly/3k6xECY.

Vieira-Neto, A. y col. (2014). «Individual and combined effects of anovulation and cytological endometritis on the reproductive performance of dairy cows». En: Journal of dairy science 97.9, 5415-5425. Online:https://bit.ly/2NigBC1.

Walsh, R. y col. (2007). «Prevalence and risk factors for postpartum anovulatory condition in dairy cows». En: Journal of dairy science 90.1, 315-324. Online:https://bit.ly/3pFKDg3.

Wathes, D. y col. (2007). «Differences between primiparous and multiparous dairy cows in the interrelation- ships between metabolic traits, milk yield and body condition score in the periparturient period.» En: Domest Anim. Endocrinol. 33.2, 203-225. Online: http:/ / bit.ly/3kcaA5L. 